\title{
Biochemical and Molecular Mechanism of Salinity Stress Tolerance in Plants
}

\author{
Vijay Kapale $^{1 *}$, Sandeep Kumar ${ }^{1}$ and Mahesh Mahajan ${ }^{2}$ \\ ${ }^{1}$ Division of Biochemistry, ICAR-Indian Agricultural Research Institute, New Delhi, India \\ ${ }^{2}$ Division of Molecular Biology and Biotechnology, ICAR-Indian Agricultural Research \\ Institute, New Delhi, India \\ *Corresponding author
}

\section{A B S T R A C T}

\section{Key w o r d s \\ ROS, SOS \\ signalling pathway, \\ Compatible solutes, \\ next-generation \\ RNA-Seq \\ technology}

\section{Article Info}

Accepted:

20 November 2018

Available Online:

10 December 2018
Salinity is one of the most aggressive environmental stresses that hamper crop productivity worldwide. Increased salt concentration in soil reduces plants ability to take up water. It also reduces nutrients availability, uptake and transport, and there distribution resulting nutritional imbalance in plant. The Salt Overly Sensitive (SOS) signalling pathway is crucial to mediate cellular signalling and to maintain ion homeostasis under salinity stress. Many HKT family plasma membrane transporters play an essential role in salt tolerance mechanism by regulating $\mathrm{Na}^{+}$and $\mathrm{K}^{+}$transportation. The nitric oxide (NO) directly or indirectly triggers numerous redox-regulated genes expression. Many genes were induced or repressed in response to salinity stress. Extensive research in the course of metabolic, cellular, and physiological analysis has elucidated that strategies or mechanisms regulating stress signaling, uptake of ions, their transport and osmotic balance, metabolism of hormone, and antioxidant play vital roles in developing plant to mitigate salt stress.

\section{Introduction}

Crop production is severely affected by high salt concentrations in soils. Salt level build up in arable soils is generally derived from irrigated water containing sodium chloride $(\mathrm{NaCl})$ and from seawater (Flowers and Yeo, 1995; Tester and Davenport., 2003). Soil salinity, on the basis of the origin of salts, can be classified into two groups; natural/primary salinity and human induced/secondary Salinity. Increased salt concentration in the soil reduces the ability of a plant to take up water. If the excessive amounts of $\mathrm{Na}^{+}$and $\mathrm{Cl}^{-}$ taken up in large amounts by roots and accumulated in leaves, then both $\mathrm{Na}+$ and $\mathrm{Cl}^{-}$ ions negatively affect plant growth by impairing metabolic processes and decreasing photosynthetic efficiency. High $\mathrm{Na}^{+}$ concentration also inhibits uptake of $\mathrm{K}^{+}$ions (Hasan et al., 2015), which results into poorer productivity and may even lead to death of plant. Thus salinity is one of the most aggressive environmental stresses that hamper crop productivity worldwide (Munns and Tester, 2008). In this review, we have 
examined the key biochemical and molecular mechanisms that can make plants tolerance to salinity stress.

\section{Biochemical mechanism of salinity stress tolerance}

Role of ion homeostasis in salinity stress tolerance

Maintaining the ion homeostasis is crucial for plant growth under normal and salinity stressed condition (Kamyab et al., 2016). At high salt concentration, the $\mathrm{Na}^{+}$ion entered in the cytoplasm is compartmentalized in to vacuole by $\mathrm{Na}^{+} / \mathrm{H}^{+}$antiporter. Increasing evidence confirmed the roles of a Salt Overly Sensitive (SOS) pathway (Figure 1) in ion homeostasis and salt tolerance (Hasegawa et $a l ., 2000)$. The Arabidopsis NADPH oxidases, AtrbohD and AtrbohF were active in ROSdependent regulation of $\mathrm{Na}^{+} / \mathrm{H}^{+}$ion homeostasis under salinity stress (Ma et al., 2012). During salinity stress, due to increased $\mathrm{Na}^{+}$ion concentration in the soil, $\mathrm{Na}^{+}$ion competes with $\mathrm{K}^{+}$ion for the same transport mechanism thereby decreasing the uptake of $\mathrm{K}^{+}$. The Arabidopsis class $1 \mathrm{HKT}$ transporters (HKT1) have been well known to cope salinity stress by preventing excess accumulation of $\mathrm{Na}^{+}$in leaves. Similar results were observed in the rice experiment in which HKT1 transporter eliminate excess $\mathrm{Na}^{+}$from xylem, thus defending the photosynthetic leaf tissues from the toxic effect of $\mathrm{Na}^{+}$(Schroeder et al., 2013). In fact more NHX isoforms e.g. LeNHX3 and LeNHX4 from tomato have been identified from different plant species and established their roles in ion $(\mathrm{Na}+\mathrm{K}+$, $\mathrm{H}+$ ) homeostasis (Galvez et al., 2012).

Role of compatible osmolytes in salinity tolerance

Compatible osmolytes (also known as compatible solutes) are a group of chemically diverse organic compounds that are low molecular weight, uncharged, polar, and highly soluble in nature and they do not interfere with the cellular metabolism even at high concentration. The proline accumulated during salt stress is not only provides salt tolerance but also serves as an organic nitrogen reserve during stress recovery. Besides these, proline plays other three major roles during stress, i.e., as a signaling molecule, as antioxidative defense molecule and a metal chelator. Both pyrroline-5carboxylate synthetase (P5CS) and pyrroline carboxylate (P5C) reductase (P5CR) enzymes are used to overproduce proline in stressed plants cell (Sairam et al., 2004). Glycine betaine as well protects the cell by osmotic adjustment, stabilizes proteins, and protects the photosynthetic apparatus from stress induced damages (Saxena et al., 2013; ChaUm et al., 2010). The salt stress induced damages were largely prevented when Oryza sativa seedlings were pretreated by glycine betaine (Rahman et al., 2002).

\section{Role of antioxidant in salinity tolerance}

Salt stress tolerance is positively correlated with accumulation of both the activity of antioxidant enzymes and the nonenzymatic antioxidant compounds (Abbasi et al., 2016). Many helicase proteins like DESD-box helicase and OsSUV3 dual helicase have been reported to improving or maintaining antioxidant machinery under stressed condition (Tuteja et al., 2013).

Exogenous application of ascorbate mitigates the adverse effects of salt stress in different plant species and promotes plant revival from the salt stress (Munir and Aftab, 2011). Another antioxidant implicated in stress mitigation is glutathione. It acts as a free radical scavenger. It also participates in the ascorbate regeneration via ascorbateglutathione cycle. Exogenously applied 
glutathione helped to maintain membrane permeability and cell viability in onion (Allium cepa) during salinity stress.

\section{Roles of polyamines in salinity tolerance}

Polyamines (PA) are ubiquitous, low molecular weight, polycationic aliphatic molecules. The putrescine (PUT), spermine (SPM) and spermidine (SPD) are the most common polyamines found within the plant (Shu et al., 2012). The PUT, which is the smallest polyamine, synthesised from either ornithine, by the action of ornithine decarboxylase (ODC) enzyme, or from arginine, by arginine decarboxylase (ADC) enzyme (Hasanuzzaman et al., 2014). When plant is exposed to salt stress, the increased level of endogenous polyamines was regulated via. Oxidative catabolism by FAD binding polyamine oxidases and copper binding diamine oxidases. Thus these oxidase enzymes play a major role in salt stress tolerance. It was observed that the mutant for polyamine biosynthetic genes, such as deficient in $A D C 1$ and $A D C 2$, is hypersensitive to salt stress (Hussain et al., 2011). Over expression of genes encoding PUT, SPD, and SPM enzymes in plants like in rice, tobacco, and Arabidopsis marks increased in polyamines level that enhanced salt tolerance in plants. SPM and SPD acts as effective inducers of Nitric oxide (NO), which is one more important signalling molecule involved in salinity tolerance

\section{Roles of nitric oxide in salinity tolerance}

Nitric oxide (NO) is an essential endogenous signaling molecule. The application of exogenous NO has been found to play important roles in stress mitigation (Sung et al., 2010). In other study it was found that the exogenous application of sodium nitroprusside, which is a NO donor, on Lupinus luteus seedlings subjected to salt stress improved seed germination and root growth. Improved plant growth under salt stress by exogenous application of NO was linked with increases in contents of the antioxidant enzymes such as SOD, APX, CAT, GPX, and GR, and decrease in malondialdehyde (MDA) production or lipid peroxidation (Nalousi et al., 2012).

High salt concentration is sensed by $\mathrm{Na}^{+}$ion sensors and induces $\mathrm{Ca}^{2+}$ and ROS. The Na+ and $\mathrm{Ca}^{2+}$ ion is sensed by $\mathrm{Ca}^{2+}$ dependent protein kinase (SOS3), which activates SOS2 kinase. Then SOS2 not only enhances ion extrusion into the apoplast by regulating $\mathrm{Na}^{+} / \mathrm{H}^{+}$antiporter (SOS1) of plasma membrane but also increase $\mathrm{Na}^{+}$ion transport into the vacuole by increasing the activity of $\mathrm{Na}^{+} / \mathrm{H}^{+}$antiporter of tonoplast. Many transcriptional factors regulate salinity stress responsive gene expression. In some plant salt content is compartmentalized in vacuole vi NHX 1.

\section{Molecular mechanism of salinity stress tolerance}

Transcriptomic analysis is widely used to screen a variety of candidate genes involved in stress responses (Wang et al., 2016). Recently, the next-generation RNA-Seq technology used to elucidate the salt-tolerance mechanism in plants like Gossypium davidsonii (Zhang et al., 2016). In Arabidopsis, salt stress moderates the upregulation of AtWRKY8 which directly binds with the $R D 29 A$ promoter, signifying it to be as one of the target genes of AtWRKY8 transcription factor (Hu et al., 2013). Wheat cultivar exposed to long-term salinity showed variation in the $b Z I P$ genes expression. The bZIP genes expression was upregulated in salt-sensitive wheat cultivar while it is decreased in salt-tolerant variety. The OsNAC5 and ZFP179 transcription factors may regulate the synthesis and accumulation of sugar, proline, and LEA proteins that 
consecutively play an integral role in stress tolerance (Rahman et al., 2002). A transcription factor in rice (Oryza sativa), SALT-RESPONSIVE ERF1 (SERF1), that showed a root specific induction upon salt and $\mathrm{H}_{2} \mathrm{O}_{2}$ treatments. Loss of SERF1 damaged the salt inducible expression of genes encoding members MAPK cascade and salt tolerance mediating TFs. Furthermore, they illustrated that SERF1 binds to the promoters of MAP3K6, MAPK5, ZFP179, and DREB2A in both in vitro and in vivo. The plants having SERF1 deficiency are more sensitive to salt stress compared with the wild type, whereas constitutive over-expression of SERF1 get better salinity tolerance. The AtMYB12 confer salt and drought tolerance through increasing the levels of flavonoids, ABA, proline, superoxide dismutase (SOD) and peroxidase (POD in transgenic Arabidopsis (Wang et al., 2016), thus AtMYB12 gene has the potential to be used to improve tolerance to salt and other abiotic stresses in plants.

High-throughput sequencing for transcriptome analysis in plant has revealed that alternative splicing affects transcriptome at higher proportion than was previously assumed. A group of Ser/Arg-rich proteins, that acts as a key regulators of alternative splicing, go through alternative splicing themselves in response to salt stress and other abiotic stresses (Staiger and Brown, 2013).

Figure.1 Signal transduction and tolerance mechanisms against salinity stress

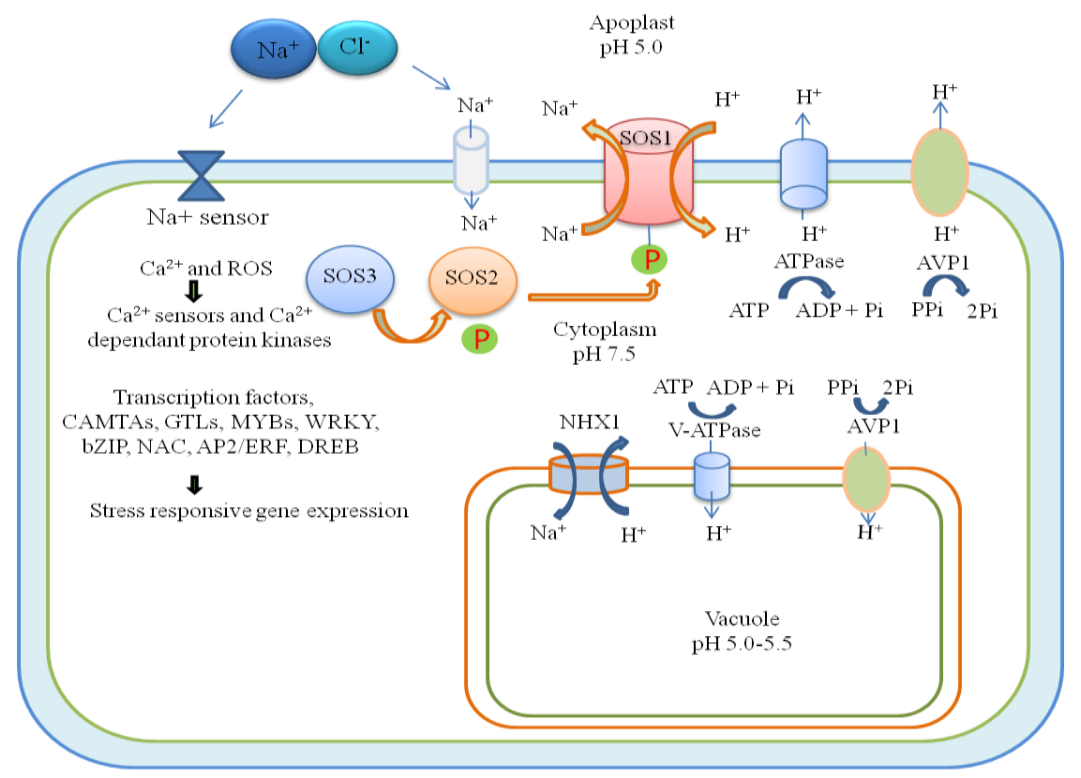

\section{Future research perspectives}

In conclusion salt stress tolerance involves a complex of responses at physiological, biochemical and molecular levels. Extensive research in the course of physiological, biochemical, molecular, and cellular analysis has elucidated that strategies or mechanisms regulating different processes, such as uptake of ions, their transport and balance, metabolism of plant hormone, antioxidant, and stress signaling, play vital roles in developing plant to mitigate salt stress. In addition, in the face of the significant advancement in the understanding of plants salt stress responses, there is still a huge gap in our understanding of ion transport across membrane, key sensor and receptor in the 
signaling transduction. Genetic engineering approaches has been proved to be an efficient way to the enlargement of salt stress-tolerant plants.

\section{References}

Abbasi, G. H., Ijaz, M. J., Akhtar, J., AnwarUl-Haq, M., Jamil, M., Ali, S., Ahmad, R., and Khan, H.N., (2016). Profiling of Anti-Oxidative Enzymes and Lipid Peroxidation in Leaves of Salt Tolerant and Salt Sensitive Maize Hybrids under $\mathrm{NaCl}$ and $\mathrm{Cd}$ Stress. Sains Malays., 45: 177-184.

Cha-Um, S., and Kirdmanee, C. (2010). Effect of glycine betaine on proline, water use, and photosynthetic efficiencies, and growth of rice seedlings under salt stress. Turk $J$. Agric. For., 34: 517-527.

Flowers, T. J., and Yeo, A. R. (1995). Breeding for salinity resistance in crop plants: where next?. Aust. J. Plant. Physiol., 22: 875-884.

Galvez, F. J., Baghour, M., Cagnac, G. H. O., Rosales, M. P. R., and Venema, K. (2012). Expression of LeNHX isoforms in response to salt stress in salt sensitive and salt tolerant tomato species. Plant Physiol. Biochem., 51: 109-115.

Hasan, A., Hafiz, H. R., Siddiqui. N., Khatun, M., Islam, R., and Mamun, A. (2015). Evaluation of wheat genotypes for salt tolerance based on some physiological traits. J. Crop Sci. Biotech., 18: 333340.

Hasanuzzaman, M., Nahar, K., and Fujita, M. (2014). Regulatory role of polyamines in growth, development and abiotic stress tolerance in plants. Plant Adaptation to Environmental Change: Significance of Amino Acids and Their Derivatives, CABI Publisher.

Hasegawa, P. M., Bressan, R. A., Zhu, J. K., and Bohnert, H. J. (2000). Plant cellular and molecular responses to high salinity. Annu. Rev. Plant Biol., 51: 463-499.

$\mathrm{Hu}$ Y, Chen L, Wang H, Zhang L, Wang F, et al. (2013). Arabidopsis transcription factor WRKY8 functions antagonistically with its interacting partner VQ9 to modulate salinity stress tolerance. Plant J., 74: 730-745.

Hussain, K., Nawaz, K., Majeed, A., Ilyas, U., Lin, F., Ali, K. and Nisar, M.F. (2011). Role of exogenous salicylic acid applications for salt tolerance in violet (Viola odorata L.). Sarhad J. Agric., 27(2): 171-175.

Kamyab, M., Kafi, M., Shahsavand, H., Goldani, M., and Shokouhifar, F. (2016). Exploring ion homeostasis and mechanism of salinity tolerance in primary tritipyrum lines (Wheat $\times$ Thinopyrum bessarabicum) in the presence of salinity. Aust. J. Crop. Sci., 10: 911-919.

Ma, L., Zhang, H., Sun, L., Jiao, Y., Zhang, G., Miao, C., and Hao, F., (2012). NADPH oxidase AtrbohD and AtrbohF function in ROS-dependent regulation of $\mathrm{Na}^{+} / \mathrm{K}^{+}$homeostasis in Arabidopsis under salt stress. J. Exp. Bot., 63: 305-317.

Munir, N., and Aftab, F. (2011). Enhancement of salt tolerance in sugarcane by ascorbic acid pretreatment. Afr. J. Biotechnol., 10: 18362-18370.

Munns, R., and Tester, M. (2008). Mechanisms of salinity tolerance. Annu. Rev. Plant Biol., 59: 651-681.

Nalousi, A. M., Ahmadiyan, S., Hatamzadeh, A., and Ghasemnezhad, M. (2012). Protective role of exogenous nitric oxide against oxidative stress induced by salt stress in bell-pepper (Capsicum annum L.). Am Eurasian J. Agric. Environ. Sci., 12: 1085-1090. 
Rahman, S., Miyake, H., and Takeoka, Y. (2002). Effects of exogenous glycine betaine on growth and ultra structure of salt-stressed rice seedlings (Oryza sativa L.). Plant Prod. Sci., 5: 33-44.

Sairam, R. K., and Tyagi, A. (2004). Physiology and molecular biology of salinity stress tolerance in plants. Current Sci., 86: 407-421.

Saxena, S. C., Kaur, H., Verma, P., Petla, B. P., Andugula, V. R. and Majee, M. (2013). Osmoprotectants: potential for crop improvement under adverse conditions, Plant Acclimation to Environmental Stress, Springer Science, New York, USA.

Schroeder JI, Delhaize E, Frommer WB, Guerinot ML, Harrison MJ, et al. (2013). Using membrane transporters to improve crops for sustainable food production. Nature, 497: 60-66

Shu, S., Guo, S. R., and Yuan, L. Y. (2012). A review: polyamines and photosynthesis. Advances in Photosynthesis-Fundamental Aspects, CBS Publishers.

Staiger, D., and Brown, J. W. (2013). Alternative splicing at the intersection of biological timing, development, and stress responses. Plant Cell, 25: 36403656.
Sung, C. H., and Hong, J. K. (2010). Sodium nitroprusside mediates seedling development and attenuation of oxidative stresses in Chinese cabbage. Plant Biotechnol. Rep., 4: 243-251.

Tester, M., and Davenport, R. (2003). $\mathrm{Na}^{+}$ tolerance and $\mathrm{Na}^{+}$transport in higher plants. Ann. Bot., 91: 503-527.

Tuteja, N., Sahoo, R. K., and Garg, B. (2013). OsSUV3 dual helicase functions in salinity stress tolerance by maintaining photosynthesis and antioxidant machinery in rice (Oryza sativa L. cv. IR64). Plant J., 76: 115127.

Wang, X., Gao, B., Liu, X., Dong, X., and Zhang, Z. (2016). Salinity stress induces the production of 2-(2phenylethyl) chromones and regulates novel classes of responsive genes involved in signal transduction in Aquilaria sinensis calli. BMC Plant Biol., 16:119.

Zhang, F., Zhu, G., Du, L., Shang, X., Cheng, C., Yang, B., Hu, Y., Cai, C. and Guo, W.(2016) Genetic regulation of salt stress tolerance revealed by RNA-Seq in cotton diploid wild species, Gossypium davidsonii, Sci. Rep., 6, 20582.

\section{How to cite this article:}

Vijay Kapale, Sandeep Kumar and Mahesh Mahajan 2018. Biochemical and Molecular Mechanism of Salinity Stress Tolerance in Plants Int.J.Curr.Microbiol.App.Sci. 7(12): 27022707. doi: https://doi.org/10.20546/ijcmas.2018.712.307 